\title{
Editorial
}

\section{SWEET - where are we heading with international type 1 diabetes registries?}

In light of the technological advances in diabetes therapy becoming more widely available and with healthcare costs rising generally, economic data regarding health care are desperately needed to allocate resources appropriately. 'SWEET' is an acronym derived from 'Better control in Pediatric and Adolescent diabeteS: Working to crEate CEnTers of Reference' and is based on a partnership of established national and European diabetes organizations (www.sweet-project.eu) led by the International Society for Pediatric and Adolescent Diabetes (ISPAD), with the initial participating pediatric centres being from the Czech Republic, France, Germany, Greece, Hungary, Italy, Luxembourg, the Netherlands, Poland, Portugal, Romania, Sweden, and the UK. In addition, the valuable contributions of the International Diabetes Federation (IDF) Europe, the Federation of European Nurses in Diabetes (FEND), and Primary Care Diabetes Europe (PCDE) need to be acknowledged. Co-funding for the initial project was granted by the European Public Health Executive Agency with additional funds from corporate partners and foundations (see acknowledgements for the complete list). The present Special Issue of Pediatric Diabetes summarizes the major findings of the project. Before SWEET, 17 out of 26 EU countries had officially recognized centers for pediatric diabetes, but only eight of them had defined criteria for becoming such a center. A system of quality control of pediatric diabetes at the national level was reported from 7/26 countries. Only 13/26 EU countries had a pediatric diabetes register. These SWEET results were based on 108 datasets from healthcare professionals caring for $>29000$ children and adolescents with diabetes in Europe.

Recommendations for diabetes care and treatment, as well as age-appropriate education for children and adolescents with diabetes and pediatric training programs for healthcare professionals have been developed by SWEET. The proposed Criteria for an European Pediatric Diabetes Reference Centre include a multidisciplinary approach, an ongoing electronic documentation of at least 150 pediatric diabetes patients $\leq 18$ years (i.e., at least age, diabetes duration, gender, $\mathrm{HbA} 1 \mathrm{c}$, type of diabetes), and the readiness to participate in external peer-reviewed auditing process and quality control circles.

An initial 12 centres were approved jointly by the ISPAD Executive Committee and IDF Europe. It has been our experience that this approval has helped to raise awareness for the efforts to achieve optimal diabetes care within the different local circumstances. There is reason to believe that the international comparison within the SWEET project will help to secure funding from payers to ensure quality delivery of care and to harmonize pediatric diabetes healthcare delivery on the highest achievable standard within the ramifications of the differing national health systems. Optimal functioning of a team requires clear identification of all team players, with the child and the family at the centre of care. So far, huge differences have been identified between the different multidisciplinary teams, not only in numbers of healthcare professionals, but as well their training and the services provided. Therefore, a Toolbox supporting the creation of Centres of Reference for pediatric and adolescent diabetes has been developed. For benchmarking and quality control, a platform was put in place that allows any participating centre to import and input data online using a standard diabetes data set for pediatric diabetes patients to monitor the long-term outcome after the conclusion of the initial SWEET project. This will allow adapting the current recommendations on the basis of the ongoing evaluations.

Adolescents with type 1 and type 2 diabetes are particularly prone to poor metabolic and psychosocial outcomes due to the potentially long diabetes duration. At the same time, the study of the effect of diabetes in this age group provides an opportunity to study early vascular ageing without other concomitant diseases. Normal ageing with diabetes starts in childhood as many processes leading to debilitating complications later in life have their onset then. This offers a unique opportunity to identify potential factors that are important to delay accelerated biological ageing 


\section{Editorial}

in diabetes. Also, preventive strategies that prove effective in childhood and adolescence are likely to be particularly cost-effective as most of the healthcare cost is incurred for the treatment of late complications.

Despite guidelines and consensus statements related to approaches, targets and therapies, there remains huge variation across EU Member States in the quantity and quality of diabetes-related clinical research and in care available for people with diabetes. This variability in research activity and service delivery is a consequence of many factors, the most significant being the social and cultural differences among countries, differences in clinical governance, and lack of structured networks of interested parties with commonly agreed goals.

Cross-sectional international analyses have repeatedly shown that the outcomes of pediatric diabetes care show considerable differences between centers that are not easily explained (1). But there is reason for an optimistic outlook. Longitudinal analyses over the years in Germany and Austria included data from 30708 patients documented in 305 diabetes centers between 1995 and 2009 (2). After multiple adjustments, mean HbAlc within this network decreased significantly by $0.038 \%$ per year. The Swedish registry also includes longitudinal data for $>10$ years (3). Although there may be a multitude of reasons for this improvement in the outcomes of pediatric diabetes, the longitudinal rather than cross-sectional electronic follow-up appears to be an important tool for local, national as well as international benchmarking and quality control circles. Thus, similar initiatives have been started elsewhere. In Kuwait, the SWEET survey was used to assess the structure of diabetes education (4). In the USA, the T1D Exchange was founded in 2010 through a grant from The Leona M. and Harry B. Helmsley Charitable Trust's Type 1 Diabetes Program (www.tldexchange.org). Individuals are being enrolled into the Registry at 67 centers throughout the USA.

The current issue of Pediatric Diabetes summarizes the outcomes of the SWEET project, and the complete SWEET reports can be found online at http://www.sweet-project.eu/index.php/countryreports/sweet-reports.

This web page also contains an email-link for reporting amendments, updates and corrections. An on-line comparison tool located at http://www.sweetproject.eu/index.php/country-reports enables one to compare parameters of any two countries. Finally, a toolbox supporting the creation of Centres of Reference for pediatric and adolescent diabetes will be put in place.

The basis for the future development of SWEET will be the electronic health record. Two electronic databases have been developed within the SWEET project for those centers not having an electronic health record of their own. The basic system DIAMAX:mini is available for free download for all ISPAD members for data collection purposes. It has been developed for all diabetes specialists who do not yet use an electronic health record and want to start electronic documentation on a limited scale. An easy export function from DIAMAX:mini into EXCEL will allow individual scientific analyses. As an ISPAD member, you receive the software free of charge by clicking on the submit button which will forward you to get your free download. You will find DIAMAX:mini on the page you first get to when logging in. If you are already logged in, go to 'Members' 'My Page'. It is possible to upgrade DIAMAX:mini to the professional diabetesoutpatient-system [DPV2] DIAMAX at any time. Compared to DIAMAX:mini several extra features are implemented in [DPV2] DIAMAX exclusively, (e.g., detailed doctor's letters, individual therapy plans, informative patient quick overviews, enhanced statistics, flexible charts, significant patient summaries, etc.). Aggregate data is de-identified and can be exported to the SWEET project from this and various other databases with SWEETconv conversion tool for longitudinal data analysis. Eventually patients will also have the ability to logon to their own secure portal to complete outcomes assessments, receive relevant reminders and education material, as well as communicate with their local pediatric diabetes treatment center. In a first step, the SWEET Online platform will allow 12 countries in several languages to connect to one unified diabetes database.

The European DIAMAP project (5) proposed a European Platform for Clinical Research in Diabetes (EPCRD) to address these issues as well as other more generic obstacles toward clinical research for the benefit of individuals with diabetes Europe. A large population of people with diabetes with variations in genetic and ethnic background could be made accessible to clinical (and basic) researchers and the sponsors of research by participation in a network with a centralized point of entry. The DIAMAP road maps have repeatedly mentioned as roadblocks the need for registries of patients, networks of specialist researchers, access to biobanks and human biological material (especially in relation to the rarer complications) and the need for more standardized treatment guidelines (Fig. 1). It was felt strongly that diabetes research would be enhanced if the clinical research community itself could drive a collaborative initiative as it deals with the consequences of research upon treatment and care delivery. Clinical research, from small studies to large-scale pharmaceutical trials, or research into health service provision is more laborious and less representative than it could be because of the number of countries, languages and organizational cultures. 


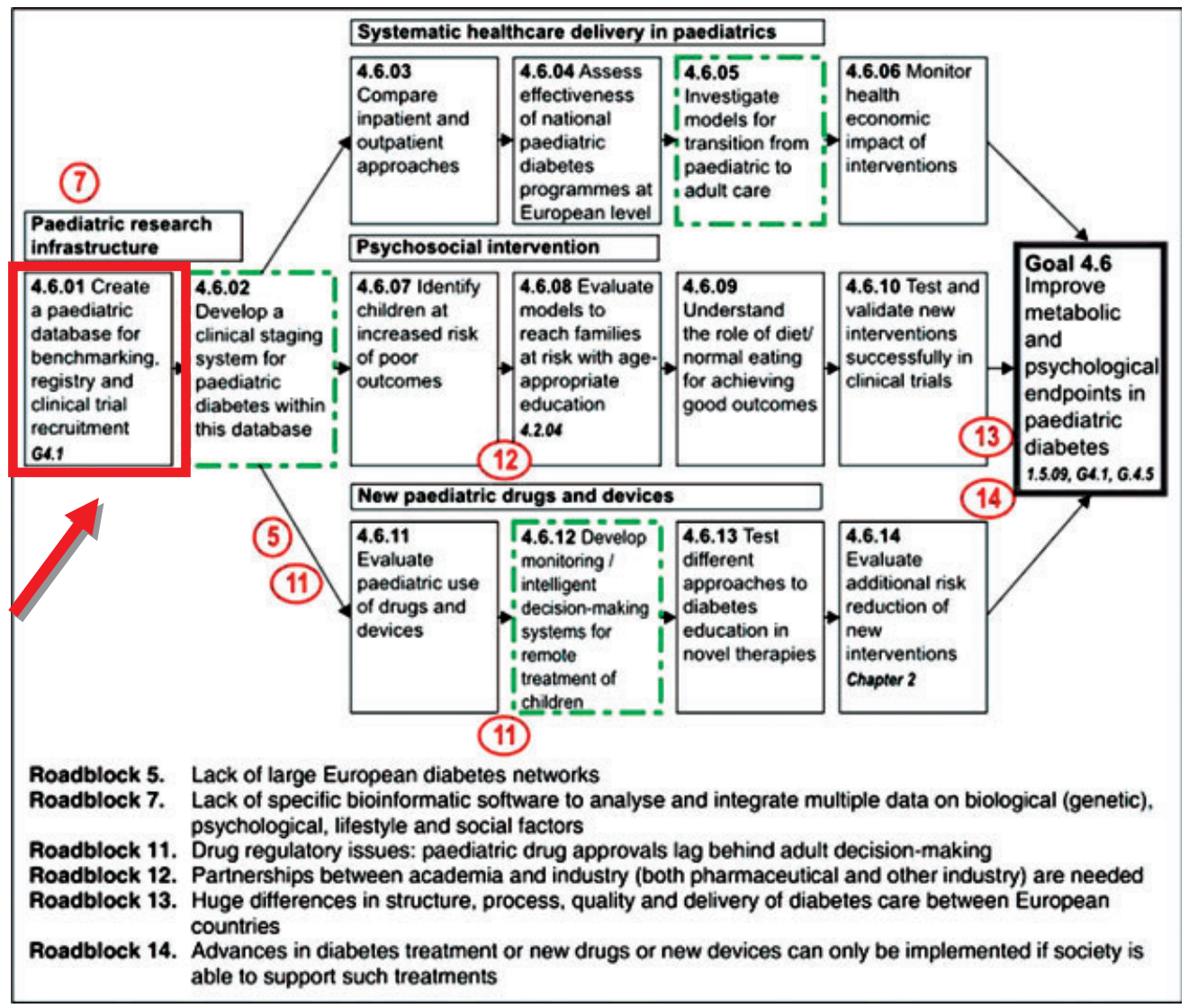

Fig. 1. DIAMAP: the pediatric roadmap for diabetes research (www.diamap.eu). Modified figure from Chapter 4. John J. Nolan (Chair), Thomas Danne, Michaela Diamant, Gillian Hood, Alexandra Kautzky-Willer, David Kerr, Asmina Mitrakou, Peter M. Nilsson, Giovanni Pacini. Clinical science and care incorporating the development of a European platform for clinical research in diabetes. The need for networking and data bases as basis for enhancing Pediatric Diabetes Research.

We believe that a collaboration of these national and international initiatives will allow a joint evaluation of these invaluable data sets. It could improve the situation for children with diabetes in Europe and beyond ensuring that all children have the right to participate fully in all the experiences of childhood and adolescence, regardless of whether they have diabetes and wherever they live. Also it could reduce the effects of accelerated vascular ageing imposed by the diabetic state and provide the basis for a long-term participation in professional and family life without or with delayed debilitating long-term diabetes complications. Under the umbrella of our scientific society ISPAD, the goal of the newly created legal entity SWEET is to continuously develop these recommendations for structure, process and outcome quality with regard to the treatment strategies and standards for pediatric diabetology, on the basis of longitudinal international patient data, as well as creating training programs for diabetes experts and patients. The SWEET project hopes to extend from the initial group of centres within countries and throughout Europe and beyond with the help of the ISPAD network.

\section{Acknowledgement}

This Special Issue of Pediatric Diabetes arises from project 2007104: 'Better control in pediatric and adolescent diabetes in the EU: working to create centres of reference (SWEET)' which has received funding from the European Union, in the framework of the Public Health Programme. The sole responsibility for the content lies with the authors. The Executive Agency is not responsible for any use that may be made of the information contained therein. The SWEET Project acknowledges with gratitude the support through unrestricted educational grants from the following Foundations and corporate sponsors (in alphabetical order): Bayer Health Care - Diabetes Care, DexCom Inc., Foundation Hannoversche Kinderheilanstalt, Johnson \& Johnson Diabetes Institute, Lilly Diabetes Excellence Centre, Medtronic Europe, The Medtronic Foundation, NovoNordisk A/S, Roche, Sanofi. The UK arm of the SWEET project has received additional funding from the Diabetes Foundation UK. This Special issue was supported through an unrestricted educational grant from Sanofi.

\section{Conflict of interest}

Thomas Danne has received honoraria for speaking engagements from several companies involved in the diabetes field. Furthermore he has received grant support from these companies (Sanofi-Aventis, Bayer, 
Roche, Johnson\&Johnson, Lilly, Medtronic, DexCom, NovoNordisk) for the conduct of studies or scientific meetings.

Riccardo Perfetti is employed by Sanofi.

Bärbel Aschemeier declares no conflict of interest.

Thomas Danne ${ }^{\mathrm{a}}$, Bärbel Aschemeier ${ }^{\mathrm{a}}$, Riccardo Perfetti $^{\mathrm{b}}$ and the SWEET group*

${ }^{a}$ Children's Hospital AUF DER BULT, D-30173,

Hannover, Germany; and ${ }^{b}$ Global Medical Affairs,

Sanofi, Diabetes Division, 75008, Paris, France e-mail: danne@hka.de

*See Appendix for representatives of the SWEET group.

\section{References}

1. Danne $\mathrm{T}$, Mortensen HB, Hougaard $\mathrm{P}$ et al. Hvidøre Study Group on Childhood Diabetes. Persistent differences among centers over 3 years in glycemic control and hypoglycemia in a study of 3,805 children and adolescents with type 1 diabetes from the Hvidøre Study Group. Diabetes Care 2001: 24: 1342-1347.

2. Rosenbauer J, Dost A, Karges B et al. DPV Initiative and the German BMBF Competence Network Diabetes Mellitus. Improved metabolic control in children and adolescents with type 1 diabetes: a trend analysis using prospective multicenter data from Germany and Austria. Diabetes Care 2012: 35: 80-86.

3. Hanberger L, Samuelsson U, Berterö C, Ludvigsson J. The influence of structure, process, and policy on $\mathrm{HbA}(1 \mathrm{c})$ levels in treatment of children and adolescents with type 1 diabetes. Diabetes Res Clin Pract 2012: 96: $331-338$.

4. Alsanaa H, Maarafie D, Alterkait N et al. Evaluation of care and education for children and young people with diabetes in Kuwait: the SWEET Project. Pediatr Diabetes 2011: 12 (Suppl. 15): 90 (Abstract).

5. Hills S, Halban PA. DIAMAP: a road map for diabetes research in Europe. J Diabetes Sci Technol 2011: 5: 794-797.

\section{Appendix}

Representatives of the SWEET group

1. Zdeněk Šumník, Ondrej Cinek, Department of Pediatrics, University Hospital Motol, Praha, Czech Republic

2. Jean-Jacques Robert, Delphine Martin, Department of Diabetes, Hôpital Necker-Enfants Malades,
Endocrinologie, Gynécologie et Diabétologie Pédiatriques, Paris, France

3. Thomas Danne, Olga Kordonouri, Bärbel Aschemeier, Jo Dalton, Susanne Klotmann, Diabetes Centre for Children and Adolescents at the Kinder- und Jugendkrankenhaus - AUF DER BULT, Hannover, Germany

4. Karin Lange, Heike Saßmann, Department of Medical Psychology, Medical School Hannover, Carl- Neuberg-Str. 1, Hannover, Germany

5. Andriana Vazeou, Center of A' Department of Pediatrics, "P\&A Kyriakou Childrens's Hospital" Athens, Greece

6. Leonardo Pinelli, Centro Regionale Specializzato di Diabetologia Pediatrica Università Verona, Italy

7. Carine de Beaufort, Michael Witsch, Department of Pediatrics, DCCP/ Pediatric Clinic $\mathrm{CH}$ de Luxembourg, Luxembourg

8. Henk-J. Veeze, Stichting Diabeter, TG Rotterdam, The Netherlands

9. Agnieszka Szypowska, Department of Diabetes at the Medical University of Warsaw, Warsaw, Poland

10. Ewa Pańkowska, Instytut Matki i Dziecka, 01-211 Warszaw, ul.M.Kasprzaka 17A, Poland

11. João Filipe Raposo, Cristina Valadas, Peadiatric Part of Associação Protectora dos Diabéticos de Portugal, Lisbon, Portugal

12. Viorel Serban, Alexandra Moisuc, Mihaela Rosu, Clinical Center 'Cristian Serban' for Evaluation and Rehabilitation for Children and Adolescents, Buziaş, Romania

13. Gun Forsander, Marie-Christine Hallmann, Department of Diabetes at the Queen Silvia Childrens Hospital Sahlgrenska University Hospital, Gothenburg, Sweden

14. Jeremy Allgrove, Barts and the London NHS Trust at the Royal London Hospital Whitechapel, London, United Kingdom

15. Sheridan Waldron, Dorset County Hospital, Dietetic Department, Dorset, United Kingdom

16. Anne Felton, FEND, 24 Holmesdale Ave, London, SW14 7BQ, United Kingdom

17. Laszlo Madacsy, First Department of Pediatrics at the Semmelweis University, Budapest, Hungary

18. Imre Rurik, Primary Care Diabetes, Europe, 4012 Debrecon, PO Box 54, Hungary

19. Frederique Duval-Pierini, L-3541 Luxembourg, Luxembourg

20. Ulrike Handke, Image - project, Munich, Germany 
Copyright of Pediatric Diabetes is the property of Wiley-Blackwell and its content may not be copied or emailed to multiple sites or posted to a listserv without the copyright holder's express written permission. However, users may print, download, or email articles for individual use. 